\title{
Flap Reconstruction and Hyperbaric Oxygen Therapy in the Management of Temporal Bone Osteoradionecrosis in an Endolymphatic Sac Tumor: Case Report
}

\author{
Daipayan Guha, M.D.(c), ${ }^{1}$ Cynthia Menard, M.D.. ${ }^{2}$ Wayne Evans, M.D., ${ }^{3}$ \\ Fred Gentili, M.D., M.Sc., ${ }^{1}$ and Gelareh Zadeh, M.D., Ph.D. ${ }^{1}$
}

Endolymphatic sac tumors (ELSTs) are rare neuroectodermal neoplasms arising within the posterior petrous bone. We present a case of a 21-year-old man who presented with a 6-month history of intermittent morning headaches, fatigue, diplopia, and gait ataxia. Imaging and surgical pathology identified an adenocarcinoma of the endolymphatic sac compressing the cerebellum and brain stem. The tumor and multiple metastases were treated with surgery, radiation, and radiosurgery. Following insertion of a ventriculoperitoneal shunt for hydrocephalus, he developed symptomatic tension pneumocephalus secondary to radionecrosis of his petrous bone, requiring flap reconstruction and use of a programmable shunt valve complemented by hyperbaric oxygen (HBO) therapy. We document here a young patient with a rare adenocarcinoma of the endolymphatic sac. This case is unique for its initial presentation without any vestibuloauditory symptoms. Metastatic spread of ELSTs is also rare. While osteoradionecrosis (ORN) of the temporal bone has been reported previously in patients with nasopharyngeal carcinoma, this is the first time it has been presented in the context of an ELST. Tension pneumocephalus is a rare complication of skull base ORN. This is the first reported use of a programmable shunt valve and HBO therapy in the management of tension pneumocephalus.

KEYWORDS: Endolymphatic sac tumor, osteoradionecrosis, hyperbaric oxygen therapy, tension pneumocephalus

The endolymphatic sac is a mucous epithelium of neuroectodermal origin lining the vestibular canal, connecting the utriculo-saccular canal to the posteromedial surface of the petrous bone. Endolymphatic sac tumors (ELSTs) are rare papillary adenocarcinomas which are locally aggressive but not known to metastasize. Patients typically present with sensorineural deafness, tinnitus, and vertigo from direct invasion of the petrous bone, and less frequently cranial nerve neuropathies from extension into the cerebellopontine
${ }^{1}$ Division of Neurosurgery, Department of Surgery, Toronto Western Hospital; Departments of ${ }^{2}$ Radiation Oncology and ${ }^{3}$ Anesthesia, Hyperbaric Oxygen Medicine Unit, University Health Network, University of Toronto, Toronto, Ontario, Canada.

Address for correspondence and reprint requests: Daipayan Guha, Toronto Western Hospital, 399 Bathurst Street, 4W-439, Toronto, ON M5T 2S8 (e-mail: deep.guha@gmail.com).
Skull Base Rep 2011;1:125-128. Copyright (C) 2011 by Thieme Medical Publishers, Inc., 333 Seventh Avenue, New York, NY 10001, USA. Tel: +1(212) 584-4662.

Received: March 25, 2011. Accepted after revision: May 9, 2011. Published online: July 21, 2011.

DOI: http://dx.doi.org/10.1055/s-0031-1284207.

ISSN 2157-6971. 
(CP) angle. A majority of ELSTs are unilateral and arise sporadically, though 11 to $30 \%$ of cases have been associated with von-Hippel Lindau (VHL) disease. Treatment is surgical and is generally curative, though recurrences have been noted due to incomplete resection.

We present here a unique case of an ELST in a young patient who presented without any vestibuloauditory symptoms. He subsequently developed diffuse intracranial metastases and drop metastases to the spine. Tay et al have previously reported on this patient in 2007, emphasizing the imaging characteristics and radiologic differential diagnoses of the primary intracranial lesion and drop spinal metastases. ${ }^{1}$ Treatment for our patient included multiple surgeries as well as radiotherapy and radiosurgery. He suffered dehiscence of his petrous bone secondary to osteoradionecrosis (ORN), resulting in a symptomatic tension pneumocephalus. Tension pneumocephalus secondary to temporal bone ORN has been reported only 3 times previously, all in the context of nasopharyngeal carcinoma. ${ }^{2-4}$ Earlier reports of tension pneumocephalus have been managed with decompression via ventricular drainage and repair of any underlying bony defects. This is the first reported use of a programmable ventriculoperitoneal (VP) shunt valve and hyperbaric oxygen (HBO) therapy in the treatment of tension pneumocephalus.

\section{CASE REPORT}

\section{History and Presentation}

A 21-year-old man of Southeast Asian descent presented with a 6-month history of intermittent morning headaches, fatigue, nausea, vomiting, and more recently diplopia. He had right-beating horizontal nystagmus and an ataxic gait, but was fully oriented with no focal motor deficits. He had no past medical conditions and was a lifetime nonsmoker and nondrinker.

\section{Interventions}

\section{FIRST OPERATION}

Computed tomography (CT) and magnetic resonance imaging (MRI) identified an intradural mass in the right posterior fossa, resulting in cerebellar and brain stem compression with associated hydrocephalus. A right suboccipital craniotomy was undertaken with the intent of tissue diagnosis and gross total resection of the $\mathrm{CP}$ angle mass. Pathology of the open tissue biopsy suggested adenocarcinoma of the endolymphatic sac. Postoperative MRI showed no residual tumor, and the patient was well aside from mild residual sensorineural hearing loss. Family history and genetic testing for VHL mutations were negative.

\section{SECOND OPERATION}

The patient again reported intermittent headaches 2.5 years after surgery. MRI demonstrated new mass lesions near the right medulla extending into the upper cervical cord. Gross total resection was again achieved, with tissue diagnosis confirming recurrence of low-grade endolymphatic sac adenocarcinoma. Salvage radiation therapy of $50 \mathrm{~Gy}$ was given to the area of recurrence as well as the original tumor site in 25 fractions.

\section{THIRD OPERATION}

The patient unfortunately suffered a third recurrence of his disease 3 years later, this time presenting with diffuse spinal metastases from T7-S1. Whole-spine radiotherapy was given with a total dose of $30 \mathrm{~Gy}$ in 10 fractions. Subsequent serial MRI demonstrated regression of the spinal metastases with no brain involvement.

The patient was well for one and a half years subsequently, until he reported progressive loss of vision in the inferior field of his right eye. MRI detected a suprasellar prechiasmatic mass abutting the right optic nerve. Surgical excision resolved the patient's visual deficit, though complete resection was not achieved on postoperative imaging. To compound matters, few months later new metastases were detected in the inferior wall of the frontal horn of the left lateral ventricle, in the inferomedial right cerebellum, and the pituitary infundibulum. Stereotactic radiotherapy of 45 Gy in 25 fractions was delivered to the suprasellar lesion and its postsurgical area. Radiosurgery was performed on the left frontal horn and right inferomedial cerebellar lesions, with radiologic stability of the lesions achieved. Nodular spinal metastases were then detected in the T10-11 and L2 regions, for which further spine radiotherapy was given.

Worsening hydrocephalus necessitated yet another surgery, this time to insert a VP shunt. Soon afterwards, the patient developed tension pneumocephalus due to osteoradionecrosis of his right petrous bone with free communication with the mastoid air cells (Fig. 1). He presented with worsening headache and nausea. This dehiscence required corrective surgery via a fascia lata graft and shunt revision to include a Codman Hakim programmable valve (Codman \& Shurtleff, Inc., Raynham, MA), and was complemented with 1 month of HBO therapy. Immediately following graft reconstruction the programmable valve was placed on its highest setting $\left(200 \mathrm{~cm} \mathrm{H}_{2} \mathrm{O}\right)$ to prevent cerebrospinal fluid (CSF) drainage and creation of a one-way valve and negative intracranial pressure. Two weeks following the surgery, when imaging confirmed no accumulation or recurrence of pneumocephalus (Fig. 2), the valve setting was decreased in two increments to allow CSF drainage at $100 \mathrm{~cm} \mathrm{H}_{2} \mathrm{O}$.

Over the 10-year course of his disease, the patient has undergone a total of six open surgeries, radiosurgery 
A
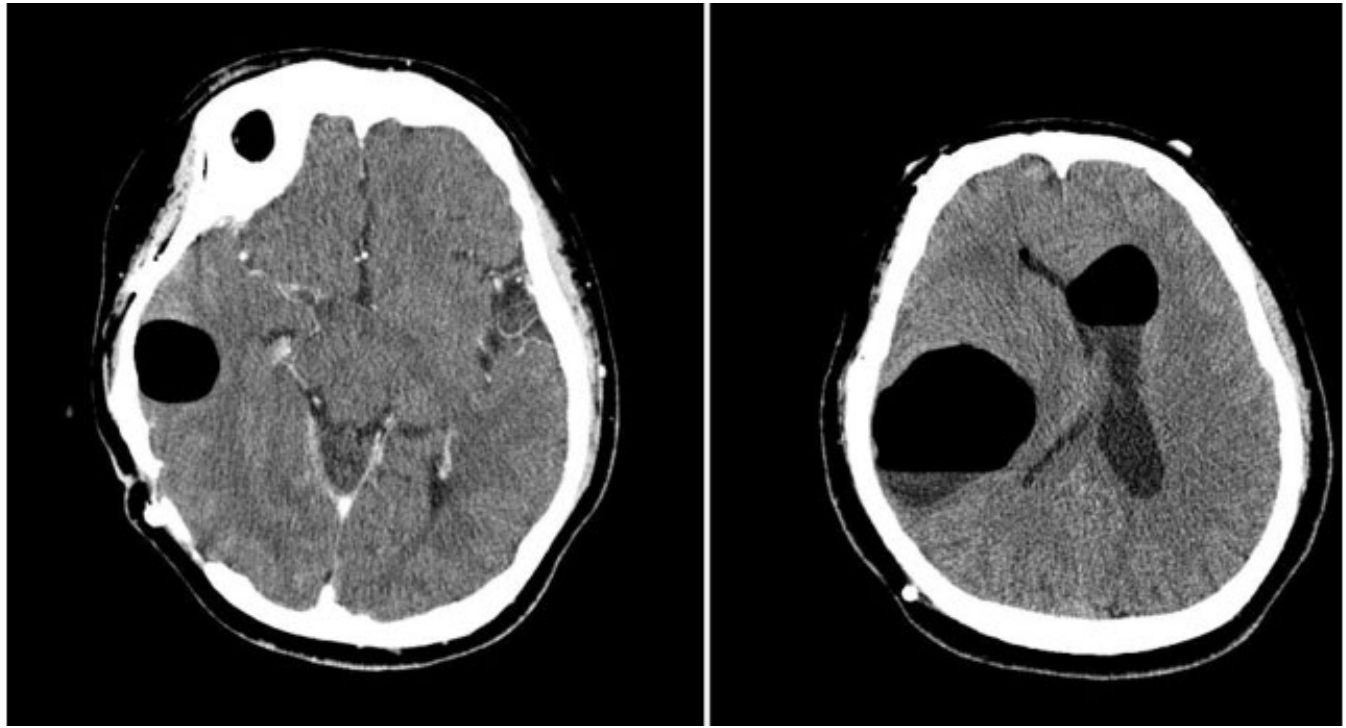

B

Figure 1 (A) Computed tomography (CT) of brain showing early development of a tension pneumocephalus following ventriculoperitoneal (VP) shunt insertion for hydrocephalus. (B) Progression to a larger symptomatic tension pneumocephalus, with visible air-fluid levels.

for three lesions, and four courses of radiotherapy to the brain and spinal cord. Total radiation dose delivered to the temporal bone was calculated by estimating the mean dose delivered to this region for each radiotherapy and radiosurgery treatment. Doses were then summed using BED (2 Gy equivalent, $\alpha / \beta=2$ ) methods, for a final total dose estimate of $140 \mathrm{~Gy}$ to the petrous bone. The patient is currently debilitated by cerebellar dysfunction stemming from his multiple therapeutic interventions in this region.

\section{DISCUSSION}

\section{ELSTs}

The endolymphatic sac is composed of three segments: a short inner portion, a highly convoluted "rugose" intermediate portion, and a posterior portion ensheathed between two layers of dura. ELSTs are rare papillary adenocarcinomas putatively arising from the rugose intermediate segment, invading aggressively into the temporal bone and adjacent $\mathrm{CP}$ angle but rarely metastasizing. ${ }^{5}$

A
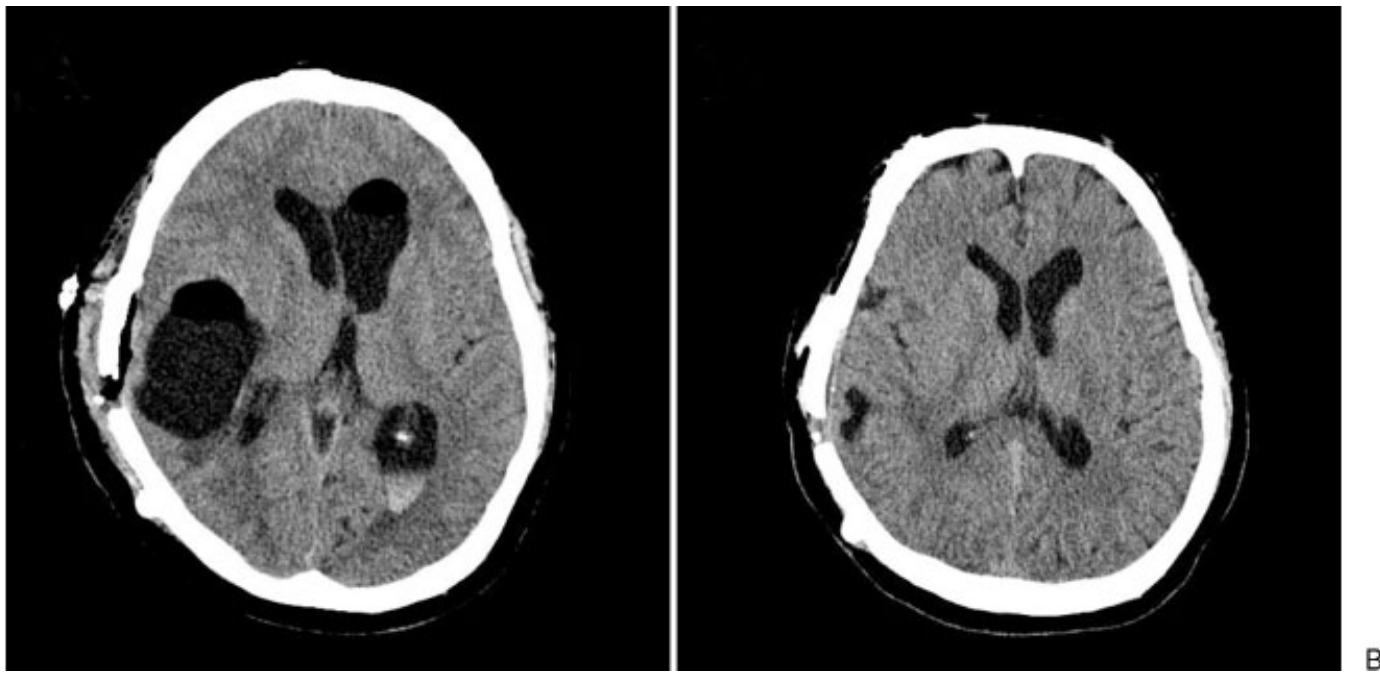

Figure 2 (A) Computed tomography (CT) of brain immediately following conversion of ventriculoperitoneal (VP) shunt to a programmable valve, demonstrating a significant reduction in intracranial air with persistent air-fluid levels. (B) CT brain 1 month following conversion to a programmable shunt valve, showing almost complete resolution of the tension pneumocephalus. 
They generally appear unilaterally and sporadically, though bilateral presentation is seen in the setting of VHL. Several instances of apparently sporadic ELSTs with mutations in the VHL gene have been reported; genetic testing for VHL is warranted in these cases, as was done for our patient.

Surgical treatment of ELSTs is usually curative, barring incomplete resection. Radiotherapy is increasingly being applied for recurrent disease or lesions not amenable to surgical excision. The role of Gamma Knife radiosurgery in treating ELSTs is not yet clear, though several cases have reported its use in treating focal recurrences. ${ }^{6}$ To our knowledge, this is the first documented case of ELST requiring multiple courses of radiosurgery for numerous focal metastases.

\section{Temporal Bone ORN}

Following a routine VP shunt insertion for hydrocephalus, our patient suffered dehiscence of his petrous bone with communication to the mastoid air cells, likely due to repeated temporal bone irradiation for numerous recurrences of his diffusely metastatic disease. Negative intracranial pressure due to CSF drainage promoted the accumulation of intracranial air, creating a one-way valve with a symptomatic tension pneumocephalus. This was corrected surgically with a fascia lata graft, followed by $\mathrm{HBO}$ therapy to promote healing. A programmable valve was employed for the VP shunt, allowing adjustment of pressure settings on follow-up visits to minimize the degree of symptomatic hydrocephalus while reducing negative intracranial pressures.

ORN is a well-established complication of radiation therapy for various malignancies, affecting up to $5 \%$ of patients. The etiology is not fully understood, but is thought to involve inflammation and fibrosis of the periosteal vessels, leading to subsequent ischemia and avascular necrosis with loss of osteocytes and osteolysis. ORN of the temporal bone has been reported extensively in the context of nasopharyngeal carcinoma and carcinomas of the external auditory canal, but never for patients with ELST or other purely intracranial disease processes, due to their largely surgical management. Temporal bone ORN may be classified as either localized or diffuse; the former is localized to the external auditory canal and typically presents with dermatitis, purulent or bloody otorrhea, otalgia, and hearing loss, while the diffuse variant extends to the skull base causing CSF otorrhea. Other potential complications of temporal bone necrosis include facial nerve palsy, meningitis, and brain abscess, as well as pneumocephalus if communication with the mastoid air cells is established as in our patient. $^{2-4}$

Treatment of ORN is difficult. Conservative therapy, involving debridement of the external auditory canal, local washing with hydrogen peroxide, and topical or oral antibiotics, has generally not been efficacious. Surgical debridement, including mastoidectomy \pm vascularized fascial flap reconstruction, is often required for definitive treatment. $\mathrm{HBO}$ therapy has long been used successfully for mandibular radionecrosis, and is increasingly being applied to other anatomic sites. ${ }^{7}$ While it has not yet been proven scientifically or in robust randomized clinical trials, it has been applied successfully in multiple head and neck malignancies, as stand-alone therapy in cases of mild radionecrosis, as an adjunct to surgery in mandibular and maxillary radionecrosis, and even as prophylaxis for patients undergoing dental extraction in a previously irradiated region. ${ }^{7}$ This is its first reported use following flap reconstruction for pneumocephalus.

\section{CONCLUSION}

ELSTs are rare adenomatous tumors of the posterior petrous bone. ELSTs have previously been considered only locally invasive; however, the possibility of both intracranial and spinal metastases should be kept in mind. Surgical resection of primary tumors is generally curative, though radiotherapy and radiosurgery may be applied for recurrent disease, metastases, or primary tumors not amenable to surgery. Tension pneumocephalus is a rare complication of temporal bone necrosis secondary to irradiation. Treatment options include surgical repair of the bone defect, ventricular decompression with a programmable valve, and HBO therapy for optimal flap healing.

\section{REFERENCES}

1. Tay KY, Yu E, Kassel E. Spinal metastasis from endolymphatic sac tumor. AJNR Am J Neuroradiol 2007;28(4): 613-614

2. Ng WF, Fung KH, Sham JS. Tension pneumocephalus-a rare complication of radiotherapy in nasopharyngeal carcinoma. Pathology 1995;27(2):204-208

3. Wu CT, Lee ST. Delayed spontaneous tension pneumocephalus caused by radionecrosis of the skull base. Br J Neurosurg 1999;13(2):214-216

4. Wang HC, Hwang JC, Peng JP, Hsieh CH, Liliang PC. Tension pneumocephalus - a rare complication of radiotherapy: a case report. J Emerg Med 2006;31(4):387-389

5. Butman JA, Kim HJ, Baggenstos M, et al. Mechanisms of morbid hearing loss associated with tumors of the endolymphatic sac in von Hippel-Lindau disease. JAMA 2007;298(1): 41-48

6. Balasubramaniam S, Deshpande RB, Misra BK. Gamma knife radiosurgery in jugular foramen endolymphatic sac adenocarcinoma. J Clin Neurosci 2009;16(5):710-711

7. Spiegelberg L, Djasim UM, van Neck HW, Wolvius EB, van der Wal KG. Hyperbaric oxygen therapy in the management of radiation-induced injury in the head and neck region: a review of the literature. J Oral Maxillofac Surg 2010; 68(8):1732-1739 